\title{
Author Correction: Single-cell chromatin state analysis with Signac
}

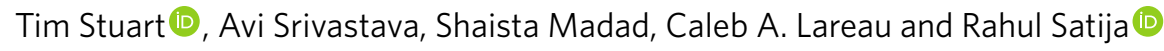

Correction to: Nature Methods https://doi.org/10.1038/s41592-021-01282-5, published online 1 November 2021.

In the version of this article initially published, there was an extraneous description in Fig. 3 caption. The text "For each box-plot, $n=6$ points (cell types)" has been removed, while the adjacent description, "For each box-plot, $n=20$ points (cell types)," has been retained.

The change has been made to the HTML and PDF versions of the article.

Published online: 7 January 2022

https://doi.org/10.1038/s41592-022-01393-7

(c) The Author(s), under exclusive licence to Springer Nature America, Inc. 2022

\section{Publisher Correction: Fast optical recording of neuronal activity by three-dimensional custom-access serial holography}

Walther Akemann, Sébastien Wolf, Vincent Villette, Benjamin Mathieu, Astou Tangara, Jozsua Fodor, Cathie Ventalon, Jean-François Léger, Stéphane Dieudonné (iD) and Laurent Bourdieu (i)

Correction to: Nature Methods https://doi.org/10.1038/s41592-021-01329-7, published online 23 December 2021.

In the version of this article initially published, several edits were omitted due to a processing error. In the Data availability section of the article and in the supplementary Nature Research Reporting Summary, the link for data used in the figures has been amended to https:// www.opendata.bio.ens.psl.eu/3D-CASH/ from ftp://ftp.biologie.ens.fr/pub/neurobiologie/3DScope/. In the original version of Extended Data Fig. 4b, the black and blue traces were truncated, and the figure has been replaced. In Extended Data Fig. 6c, a color bar has been restored above the Trial \#3 data. The changes have been made to the Supplementary Information and HTML and PDF versions of the article.

Published online: 25 January 2022

https://doi.org/10.1038/s41592-022-01401-w

(๑) The Author(s), under exclusive licence to Springer Nature America, Inc. 2022 